\title{
Early Clinical PET Imaging Results with the Novel PHF-Tau Radioligand [F18]-T808
}

\author{
David T. Chien a , A. Katrin Szardenings ${ }^{\mathrm{a}}$, Shadfar Bahri ${ }^{\mathrm{b}}$, Joseph C. Walsh ${ }^{\mathrm{a}}$, Fanrong Mu ${ }^{\mathrm{a}}$, \\ Chunfang Xia ${ }^{a}$, William R. Shankle ${ }^{c}$, Alan J. Lerner ${ }^{\mathrm{d}}$, Min-Ying Su ${ }^{\mathrm{b}}$, Arkadij Elizarov ${ }^{\mathrm{a}}$ \\ and Hartmuth C. Kolb ${ }^{\mathrm{a}, *}$ \\ ${ }^{a}$ Siemens Molecular Imaging, Inc., Culver City, CA, USA \\ ${ }^{\mathrm{b}}$ Tu and Yuen Center for Functional Onco-Imaging, Department of Radiological Sciences, University of California \\ Irvine, CA, USA \\ ${ }^{\mathrm{c}}$ Shankle Clinic Memory and Cognitive Disorders Program, Hoag Neurosciences Institute, Newport Beach, \\ CA, USA \\ ${ }^{\mathrm{d}}$ University Hospitals - Case Medical Center, Department of Neurology, Cleveland, OH, USA
}

Accepted 1 July 2013

\begin{abstract}
Aggregates of hyperphosphorylated tau (PHF-tau), such as neurofibrillary tangles, are linked to the degree of cognitive impairment in Alzheimer's disease. We have recently reported early clinical results of a novel PHF-tau targeting PET imaging agent, [F18]-T807. Since then, we have investigated a second novel PHF-tau targeting PET imaging agent, [F18]-T808, with different pharmacokinetic characteristics, which may be favorable for imaging Alzheimer's disease and other tauopathies. Here, we describe the first human brain images with [F18]-T808.
\end{abstract}

Keywords: Alzheimer's disease, amyloid- $\beta$, brain imaging, mild cognitive impairment, molecular imaging, neurofibrillary tangles, PHF-tau, tau, tau aggregates, tauopathy

\section{INTRODUCTION}

Alzheimer's disease (AD) has a widespread and burdensome impact on patients, their family members, caregivers, and the healthcare system. Currently, there are five drugs approved by the United States Food and Drug Administration (FDA) that treat the clinical symptoms of $\mathrm{AD}$, but unfortunately not the underlying causative pathology responsible for the onset and continuation of AD [1]. New research aims to directly modify the disease progression by targeting the formation of the pathological elements of $\mathrm{AD}$, amyloid plaques and insoluble paired helical filaments of tau (PHF-tau). Aggregation of PHF-tau leads to the formation of neurotoxic neurofibrillary tangles (NFTs).

\footnotetext{
*Correspondence to: Hartmuth C. Kolb, Siemens Molecular Imaging, Inc., 6100 Bristol Parkway, Culver City, CA 90230, USA. Tel.: +1 310804 7324; Fax: +1 310744 4879; E-mail: hckolb@ me.com.
}

Both PHF-tau and NFTs disrupt normal neuronal function and lead to neuronal death, resulting in cognitive decline over time. Since the formation of these PHF-tau aggregates may precede the cognitive symptomology of AD by decades, PHF-tau may be a useful pre-symptomatic biomarker of AD. Furthermore, the density and distribution of PHF-tau in the brains of $\mathrm{AD}$ patients better correlates with the severity of cognitive impairment than that of $A \beta$ plaques $[2,3]$. This promising link between pathology and disease intensity provides an opportunity for a biomarker approach to more accurately assess disease burden. A reliable diagnostic tool for PHF-tau could enable the diagnosis of $\mathrm{AD}$ years prior to symptom presentation, objectively quantify disease progression, and accelerate the discovery of effective treatments.

Positron emission tomography (PET) allows the non-invasive detection and quantification of proteins linked to disease. PET may have advantages over anatomical imaging (CT, MRI) or invasive techniques, 
such as cerebrospinal fluid (CSF) sampling, since anatomical structural changes often occur after molecular changes have already manifested themselves and CSF sampling necessitates an invasive lumbar puncture procedure. Currently, most of the AD-targeting PET radiotracers focus on amyloid plaques, including [F18]-florbetapir (Amyvid ${ }^{\circledR}$ ), approved by the FDA in 2012. There are few PET imaging radiotracers in clinical development for detecting pathological tau in human subjects, including THK523 [4-7] and our [F18]-T807 and [F18]-T808. Recently, we have reported initial clinical results of a first-in-man study with the novel PHF-tau PET radiotracer [F18]-T807 [8]. In this study, PET images from the first 6 subjects displayed favorable imaging kinetics, low non-specific binding of the radiotracer, high target cortical to cerebellum ratios, and an accumulation pattern in key brain regions which mirrored the current understanding of tau deposition in AD patients $[9,10]$. Our second PET imaging agent, [F18]-T808, showed similar tau affinity and selectivity in in vitro and in vivo preclinical studies, but displayed faster pharmacokinetics than [F18]-T807 in rodents [11]. The radiotracer's selectivity of PHFtau over $A \beta$ plaque was $>27$ fold as measured by an autoradiography comparison of human cortical brain sections of varying $A \beta$ and tau deposition. In addition, [F18]-T808 has little white matter binding in the same autoradiography assay, with non-detectable or minimal binding toward off-target proteins such as MAO, or other brain receptors. Preclinical imaging in mice demonstrated suitable brain uptake and washout profiles consistent with the time needed for PET imaging acquisition in humans. However, in contrast to [F18]-T807, [F18]-T808 underwent slow defluorination in rodents. At the outset of this study, it was not clear how fast defluorination would be in humans and whether it would compromise the ability to quantify the radiotracer's uptake in key brain regions. Thus, the radiotracer was further investigated for imaging NFTs in $\mathrm{AD}$ subjects.

Herein, we report the initial [F18]-T808 PET imaging results obtained with eleven human subjects enrolled in the [F18]-T808 first-in-man clinical trial.

\section{MATERIALS AND METHODS}

Eleven human subjects ( 8 subjects in the AD group and 3 subjects in the healthy control (HC) group) have received the investigational product [F18]-T808 and completed the study protocol. Four additional subjects enrolled in the trial did not complete the study proto- col. All participants met the inclusion and exclusion criteria set forth in Supplementary Table 1. For the patients in the $\mathrm{AD}$ group, the treating neurologists had previously made the clinical diagnosis of $\mathrm{AD}$. The AD subjects (\#0405, \#0406, \#0407, \#0410, \#0411, \#0412, \#0413, \#0414) had a mean Mini Mental State Examination (MMSE) score of 18 and HC subjects (\#0401, \#0402, \#0404) had a mean score of 30 [12]. Dynamic PET scans from 0 to $60 \mathrm{~min}$ and a static scan from 80 to $100 \mathrm{~min}$, as well as CT scans, were acquired for each subject. Volume of interest (VOI) regions were manually drawn on the CT scans for the gray matter in various brain regions, including the cerebellum. The VOIs on the CT scans were then applied to the PET scans. For each VOI, the standard uptake value (SUV) and SUV ratio to the cerebellum (SUVR) were calculated.

Both the FDA and the participating Institutional Review Board approved the study protocol and informed consent. All participants, or their appointed representative, reviewed and signed the informed consent form.

\section{Radiosynthesis}

Clinical doses of [F18]-T808 were prepared by Siemens Molecular Imaging Biomarker Research (Culver City, CA). The radiolabeling of [F18]-T808 was readily accomplished on an automated Explora ${ }^{\circledR}$ $R N$ radiosynthesis module with a total synthesis time of less than $90 \mathrm{~min}$.

Briefly, [O18]-water was bombarded in a Siemens RDS-111 cyclotron to afford approximately $2 \mathrm{Ci}$ of aqueous [F18]-fluoride. The [F18]-fluoride was then azeotropically dried with acetonitrile under reduced pressure and a stream of argon gas. The anhydrous [F18]-fluoride was reacted with the T808 precursor in the presence of DMSO, $\mathrm{K}_{2} \mathrm{CO}_{3}$, and $\mathrm{K} 222$ at $90^{\circ} \mathrm{C}$ for $5 \mathrm{~min}$. The crude reaction mixture was briefly cooled to $55^{\circ} \mathrm{C}$ before being treated with $3 \mathrm{~N} \mathrm{NaOH}$ and subsequently heated at $100^{\circ} \mathrm{C}$ for $10 \mathrm{~min}$ (Fig. 1). This base-mediated decomposition of unreacted T808 precursor was necessary to prevent co-elution of the precursor with the product during the semi-preparation HPLC purification. The crude reaction mixture was purified using $25 \%$ acetonitrile/75\% $20 \mathrm{mM}$ sodium phosphate buffer. The desired HPLC fraction was collected and reformulated using a C18 sep-pack to afford a final formulation of [F18]-T808 in 10\% ethanol: $90 \%$ water containing $10 \mathrm{mg} / \mathrm{mL}$ of ascorbic acid, all of which was sterile filtered prior to dose dispensing. The doses of [F18]-T808 for this study were 
synthesized with an average yield of $37 \%$ (decay corrected, $n=6$ ) with specific activity values ranging from 4.7 to $11.95 \mathrm{Ci} / \mu \mathrm{mol}$ at the end of the synthesis.

\section{PET/CT scans}

The PET/CT scanner (GE Discovery VCT) was validated using the SNMMI's Clinical Trials Network brain phantom prior to subject enrollment.

The subjects were placed supine on the camera table with their head secured firmly with fixation straps. Attenuation correction CT was performed using a 64slice multidetector helical scanner prior to each of the two PET acquisition sessions. The CT parameters were $\mathrm{kVp}$ of $120, \mathrm{~mA}$ of 100 , rotation cycle of $0.5 \mathrm{~s}$, pitch of 0.984:1, and reconstructed slice thickness of $3.75 \mathrm{~mm}$. All subjects received a single intravenous bolus of approximately $370 \mathrm{MBq}(10 \mathrm{mCi})$ of [F18]-T808. The radiotracer was infused over $20 \mathrm{~s}$ while dynamic PET acquisition was simultaneously started. The first PET acquisition timeframe from 0 to $60 \mathrm{~min}$ was binned into 38 dynamic frames $(10$ frames $\times 6 \mathrm{~s}, 6$ frames $\times 20 \mathrm{~s}$, 4 frames $\times 30 \mathrm{~s}, 5$ frames $\times 60 \mathrm{~s}, 5$ frames $\times 120 \mathrm{~s}, 8$ frames $\times 300 \mathrm{~s}$ ).

The subjects were given a $15-\mathrm{min}$ break followed by a second attenuation correction CT and PET imaging session acquired from 80 to $100 \mathrm{~min}$ post injection and summed into one static frame.

All PET imaging data were acquired in a single bed position with 3-dimensional list mode. PET reconstruction parameters used were matrix size of $128 \times 128$, FORE-Iterative algorithm, reconstruction diameter of $25.6 \mathrm{~cm}$; iterations of 7 , subsets of 21, loop filter of $2.0 \mathrm{~mm}$, and no Z-axis filter.

\section{Image analysis}

DICOM image data were reviewed on an Inveon Research Workplace (Edition 4.0.0.3, Siemens). The image reviewer was unblinded to the subject demographics or diagnosis. Sample VOIs for analysis were defined by manual contouring of the anatomical structures on corresponding $\mathrm{CT}$ images and the reviewer was blinded to the PET images (Supplementary Figure 2). VOIs were created for the gray matters of the frontal lobes, parietal lobes, lateral temporal lobes, mesial temporal lobes, occipital lobes, and cerebellum, as well as the genu region of the white matter and the approximate area of the hippocampus. Anatomical landmarks used to contour the VOIs included, all sulci, fissures, and ventricles (see Supplementary Material for details). The VOIs contoured on CT were applied to each dynamic frame of the first PET emission images for dynamic analysis and the second PET emission images for retention analysis of AD and HC subjects. The mean SUVs were measured based on these VOIs and SUVRs (relative to the cerebellum) were calculated as the ratios of (SUV mean of target VOI)/(SUV mean of cerebellum VOI). No partial-volume corrections were performed.

\section{RESULTS}

\section{Subjects}

The characteristics of the 3 subjects in the $\mathrm{HC}$ group and 8 subjects in the AD group are summarized in Table 1 . The mean radioactivity injected was $372 \mathrm{MBq}(10.1 \mathrm{mCi})$ for the $\mathrm{AD}$ group and $379 \mathrm{MBq}(10.2 \mathrm{mCi})$ for the HC group. The MMSE score of each of the three subjects in the HC group (\#0401, \#0402, \#0404) was 30. In the AD group, subjects \#0410 (MMSE= 23), \#0411 (MMSE = 24), \#0412 (MMSE = 24), and \#0413 (MMSE=24) had mild dementia, while subjects \#0405 (MMSE = 12), \#0406 (MMSE = 14), \#0414 (MMSE = 19), and \#0407 $(\mathrm{MMSE}=3)$ had moderate or severe dementia. In addition to the eleven subjects that completed the study protocol, there were three subjects (\#0403, \#0408, \#0409) that withdrew prior to receiving the investigational product. Another subject (\#0601) received the investigational product but did not complete the study protocol. This subject was found to be anemic at the screening visit and became agitated at the second visit, which prevented the completion of the PET scan. These were reported to the FDA as separate adverse events that the investigator deemed unrelated to the investigational product.

\section{Dynamic imaging analysis}

PET/CT imaging and radiotracer infusion was started simultaneously for all subjects, except for HC subject \#0404 due to technical reasons. For the latter subject, a review of the first frame $(0-6 \mathrm{~s})$ of the dynamic images revealed an outlying cerebellum SUV (1.70) that was far above all other subjects, which had cerebellum SUVs of $\leq 0.05$ (range $=0.01-0.05$ ). Because of this technical failure, subject \#0404 was excluded from the kinetic analysis.

The SUV time activity curves (TACs) for the 0 to 60 min dynamic images showed the radiotracer's rapid distribution throughout the brains of all subjects in both the AD and HC group (Fig. 2). The highest SUV 
Table 1

Patient demographics

\begin{tabular}{|c|c|c|c|c|c|c|c|c|c|c|c|}
\hline \multirow[t]{2}{*}{ Subject \# } & \multicolumn{3}{|c|}{ Healthy control group } & \multicolumn{8}{|c|}{ Alzheimer's disease group } \\
\hline & $\# 0401$ & $\# 0402$ & $\# 0404$ & $\# 0405$ & \#0406 & $\# 0407$ & $\# 0410$ & $\# 0411$ & $\# 0412$ & $\# 0413$ & $\# 0414$ \\
\hline MMSE & 30 & 30 & 30 & 12 & 14 & 3 & 23 & 24 & 24 & 24 & 19 \\
\hline Age (years old) & 81 & 61 & 70 & 96 & 55 & 69 & 80 & 66 & 69 & 77 & 65 \\
\hline Education (years) & 20 & 15 & 14 & 14 & 13 & 16 & 18 & 17 & 13 & 18 & 16 \\
\hline Gender (M/F) & M & $\mathrm{F}$ & $\mathrm{F}$ & $\mathrm{F}$ & M & $\mathrm{F}$ & $\mathrm{F}$ & M & M & M & $\mathrm{F}$ \\
\hline Height (meters) & 1.68 & 1.57 & 1.68 & 1.52 & 1.73 & 1.6 & 1.62 & 1.75 & 1.91 & 2.01 & 1.55 \\
\hline Weight (kg) & 59 & 54 & 73 & 59 & 68 & 62 & 54 & 73 & 88 & 81 & 57 \\
\hline Dose $(\mathrm{MBq})$ & 386 & 396 & 354 & 359 & 368 & 394 & 356 & 400 & 371 & 373 & 355 \\
\hline
\end{tabular}

Table 2

[F18]-T808 standardized uptake values (SUVs) mean and target-to-cerebellum relative SUVs (SUVRs) from 30 to 50 min post injection were measured in the cerebellum, white matter, mesial temporal, lateral temporal, parietal, frontal, and occipital lobes, and approximate area of the hippocampus

\begin{tabular}{|c|c|c|c|c|c|c|c|c|c|c|c|}
\hline \multirow[t]{2}{*}{ Subject \# } & \multicolumn{3}{|c|}{ Healthy control group } & \multicolumn{8}{|c|}{ Alzheimer's disease group } \\
\hline & $\# 0401$ & $\# 0402$ & $\# 0404$ & $\# 0405$ & $\# 0406$ & $\# 0407$ & $\# 0410$ & $\# 0411$ & $\# 0412$ & $\# 0413$ & $\# 0414$ \\
\hline MMSE & 30 & 30 & 30 & 12 & 14 & 3 & 23 & 24 & 24 & 24 & 19 \\
\hline \multicolumn{12}{|c|}{ SUV mean } \\
\hline Cerebellum & 0.77 & 0.64 & 0.72 & 1.30 & 0.57 & 0.83 & 0.78 & 0.74 & 0.68 & 0.66 & 0.75 \\
\hline White matter & 0.51 & 0.52 & 0.56 & 0.97 & 0.50 & 0.66 & 0.60 & 0.59 & 0.51 & 0.53 & 0.60 \\
\hline Mesial temporal lobe & 0.70 & 0.64 & 0.69 & 1.20 & 0.73 & 1.10 & 0.84 & 0.94 & 0.75 & 0.80 & 0.95 \\
\hline Lateral temporal lobe & 0.74 & 0.67 & 0.67 & 1.10 & 0.94 & 1.40 & 0.99 & 1.10 & 0.79 & 0.86 & 1.10 \\
\hline Parietal lobe & 0.69 & 0.65 & 0.67 & 1.20 & 0.92 & 1.40 & 0.92 & 1.10 & 0.73 & 0.68 & 1.10 \\
\hline Frontal lobe & 0.64 & 0.66 & 0.68 & 1.10 & 0.86 & 1.20 & 0.77 & 0.80 & 0.66 & 0.63 & 1.00 \\
\hline Occipital lobe & 0.72 & 0.65 & 0.72 & 1.30 & 0.67 & 1.10 & 0.77 & 0.78 & 0.71 & 0.76 & 0.90 \\
\hline \multicolumn{12}{|l|}{ Approximate area of the } \\
\hline hippocampus & 0.79 & 0.68 & 0.69 & 1.40 & 0.70 & 1.10 & 0.90 & 0.98 & 0.87 & 0.79 & 0.98 \\
\hline \multicolumn{12}{|c|}{ Target to cerebellum SUV ratio } \\
\hline Cerebellum & 1.00 & 1.00 & 1.00 & 1.00 & 1.00 & 1.00 & 1.00 & 1.00 & 1.00 & 1.00 & 1.00 \\
\hline White matter & 0.66 & 0.82 & 0.78 & 0.75 & 1.11 & 0.80 & 0.77 & 0.80 & 0.76 & 0.80 & 0.80 \\
\hline Mesial temporal lobe & 0.90 & 1.01 & 0.96 & 0.92 & 1.28 & 1.32 & 1.08 & 1.27 & 1.12 & 1.21 & 1.27 \\
\hline Lateral temporal lobe & 0.96 & 1.04 & 0.94 & 0.85 & 1.64 & 1.68 & 1.27 & 1.49 & 1.16 & 1.30 & 1.47 \\
\hline Parietal lobe & 0.89 & 1.02 & 0.93 & 0.92 & 1.60 & 1.68 & 1.18 & 1.49 & 1.08 & 1.03 & 1.47 \\
\hline Frontal lobe & 0.82 & 1.03 & 0.94 & 0.85 & 1.50 & 1.44 & 0.98 & 1.09 & 0.97 & 0.96 & 1.33 \\
\hline Occipital lobe & 0.93 & 1.01 & 1.00 & 1.00 & 1.16 & 1.32 & 0.99 & 1.06 & 1.05 & 1.15 & 1.20 \\
\hline \multicolumn{12}{|l|}{ Approximate area of the } \\
\hline hippocampus & 1.02 & 1.06 & 0.96 & 1.08 & 1.22 & 1.32 & 1.15 & 1.32 & 1.28 & 1.20 & 1.31 \\
\hline
\end{tabular}<smiles>COCCC1CCN(c2ccn3c(n2)nc2ccccc23)CC1</smiles>

T808 precursor a) $\mathrm{K}[\mathrm{F}-18] \mathrm{F}, \mathrm{K} 222, \mathrm{~K}_{2} \mathrm{CO}_{3}$ $\mathrm{DMSO}, 90^{\circ} \mathrm{C}, 5 \mathrm{~min}$

b) $\mathrm{NaOH}, 100^{\circ} \mathrm{C}, 10 \mathrm{~min}$

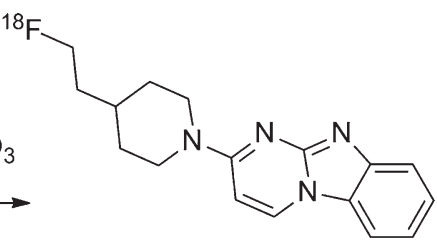

[F-18]-T808

Fig. 1. Radiosynthesis of [F18]-T808.

in the cerebellum was reached between $0.5-3.0 \mathrm{~min}$. [F18]-T808 cleared rapidly from the cerebellum with less than half of the maximum activity remaining after $16.2 \mathrm{~min}$ (range $=10-30 \mathrm{~min}$ ). For subjects in the $\mathrm{HC}$ group, [F18]-T808 also cleared rapidly from the cortical regions at the same rate as the cerebellum. For the 96-year-old subject \#0405, who displayed signs of dementia (MMSE = 12), a similar pattern of radiotracer clearance as for the subjects in the HC group was observed. In contrast, the radioactivity in all 7 other younger dementia subjects, cleared slower from the cortical regions relative to the cerebellum, resulting in a selective retention in these cortical regions where PHF-tau accumulation is expected for AD 

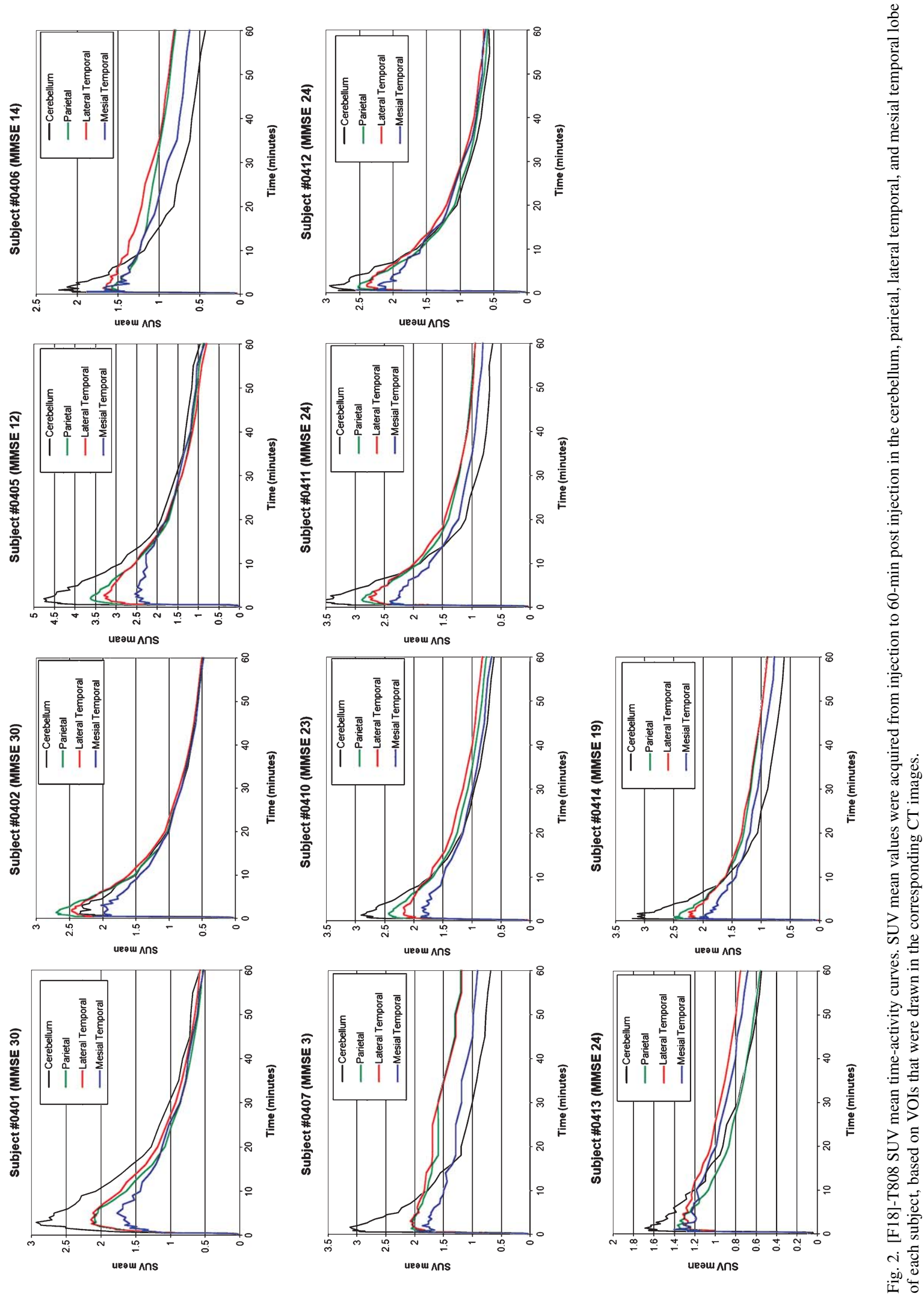
Table 3

[F18]-T808 standardized uptake values (SUVs) mean and target-to-cerebellum relative SUVs (SUVRs) from 80 to 100 min post injection were measured in the cerebellum, white matter, mesial temporal, lateral temporal, parietal, frontal, and occipital lobes, and approximate area of the hippocampus

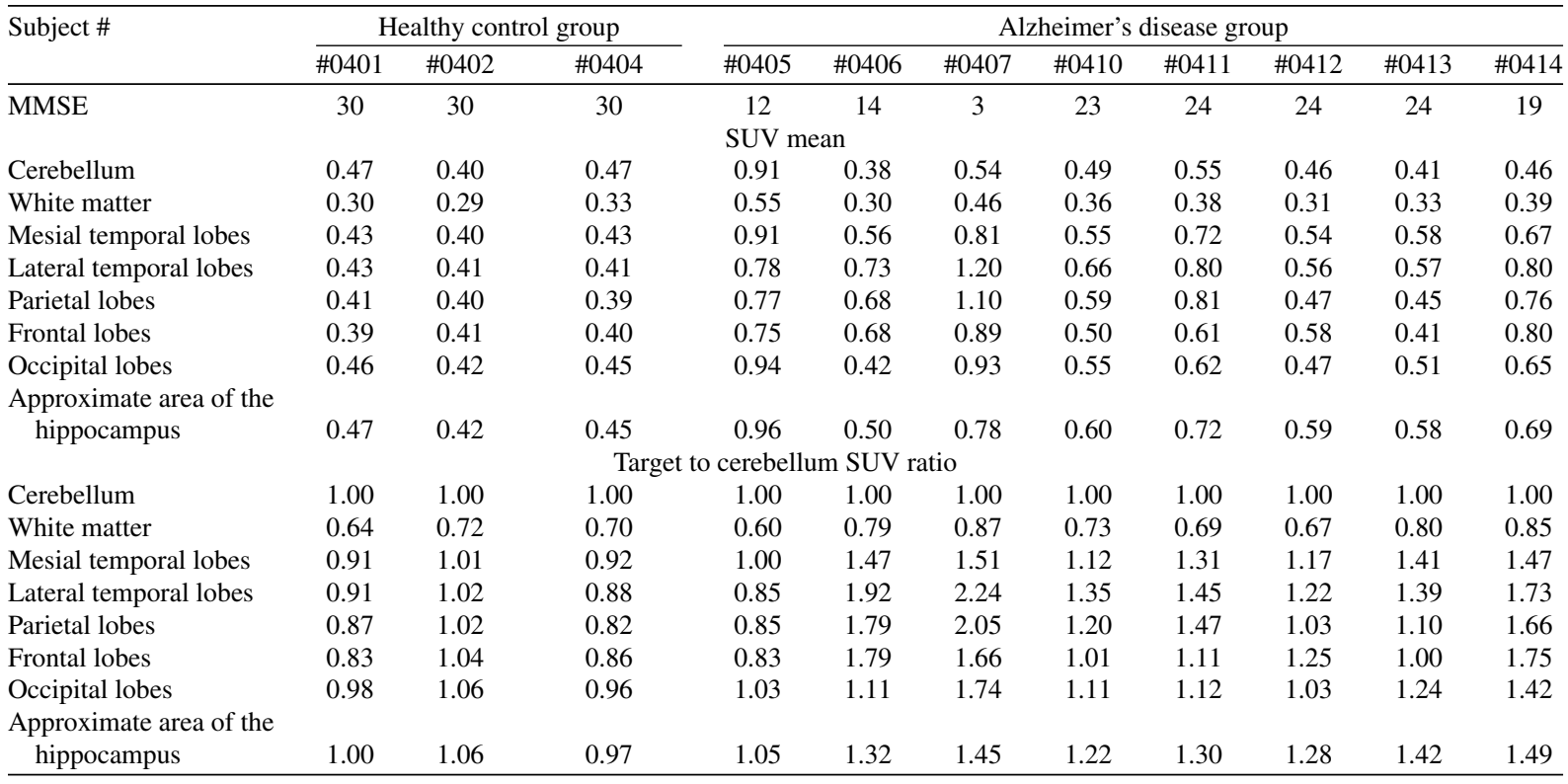

subjects $[9,10]$. The retention was seen at an early time point with the SUVR greater than 1.0 in the lateral temporal lobes of $\mathrm{AD}$ subjects starting at 7 to $10 \mathrm{~min}$ post injection.

The cortical-to-cerebellum SUVR TAC in the 0 to 60 min dynamic images showed that subjects in the HC group had SUVRs of $\leq 1.1$ for all cortical regions after the first 3 min of initial perfusion, consistent with a pattern of lowered radiotracer retention (Fig. 3). A similar pattern was seen for subject \#0405. In comparison, the other AD subjects demonstrated a continually increasing SUVR in all cortical regions while the radiotracer continued to washout from the cerebellum indicative of selective radiotracer retention.

\section{Static imaging analysis}

30 to 50 minutes summed images (Figure 4, Table 2)

Because there was rapid radiotracer washout from reference tissue, we examined the potential of analyzing the differentiation between subjects in the $\mathrm{AD}$ and $\mathrm{HC}$ groups at an early time point, 30-min post injection. Thus, the 30 to $50 \mathrm{~min}$ dynamic frames were summed into static images and analyzed. There was low [F18]-T808 retention for all cortical regions with SUVR values below 1.10 for the $\mathrm{HC}$ subjects and subject \#0405 (MMSE = 12).
In contrast, the subjects with mild $\mathrm{AD}$ and MMSE scores of 23-24 (subjects \#0410, \#4011, \#4012, \#0413) demonstrated a range of mild to moderate levels of radiotracer retention in several targeted cortical regions, including the hippocampal area $(\mathrm{SUVR}=1.15-1.32)$, mesial temporal lobe $(\mathrm{SUVR}=1.08-1.27)$, lateral temporal lobe $(\mathrm{SUVR}=1.16-1.49)$, and parietal lobe (SUVR = 1.03-1.49). However, none of these subjects had increased radiotracer retention in the frontal lobe (SUVR <1.10).

Comparatively, subjects with moderate or severe $\mathrm{AD}$ retained increased levels of the radiotracer in the frontal lobe. For subject \#0414 (MMSE = 19), the highest level of retention was in both the parietal lobe $(\mathrm{SUVR}=1.47)$ and lateral temporal lobe $(\mathrm{SUVR}=1.47)$, followed by the frontal lobe $($ SUVR $=1.33)$, the hippocampal area $(\mathrm{SUVR}=1.31)$, and mesial temporal lobe $(\mathrm{SUVR}=1.27)$. For subjects with increasingly severe $\mathrm{AD}$, the radiotracer uptake resulted in the same pattern of retention but with increased signal intensity. Subjects \#0406 $(\mathrm{MMSE}=14)$ and \#0407 (MMSE = 3) had increased radiotracer retention in the lateral temporal lobe (SUVR $=1.64$ and 1.68, respectively) and parietal lobe (SUVR $=1.60$ and 1.68 , respectively), followed by the frontal lobe (SUVR $=1.50$ and 1.44 , respectively), the mesial temporal lobe $(\mathrm{SUVR}=1.28$ and 1.32 , 

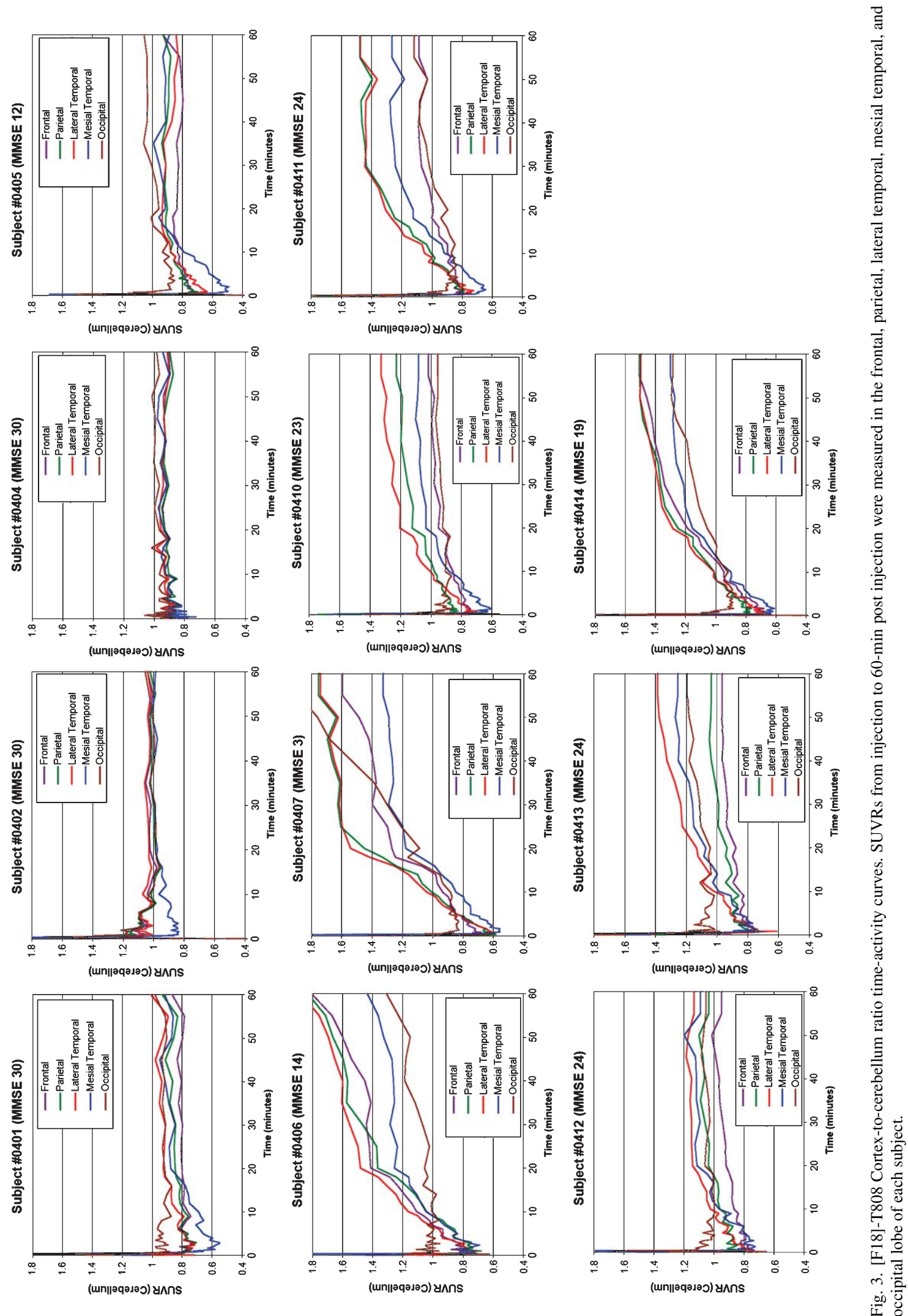

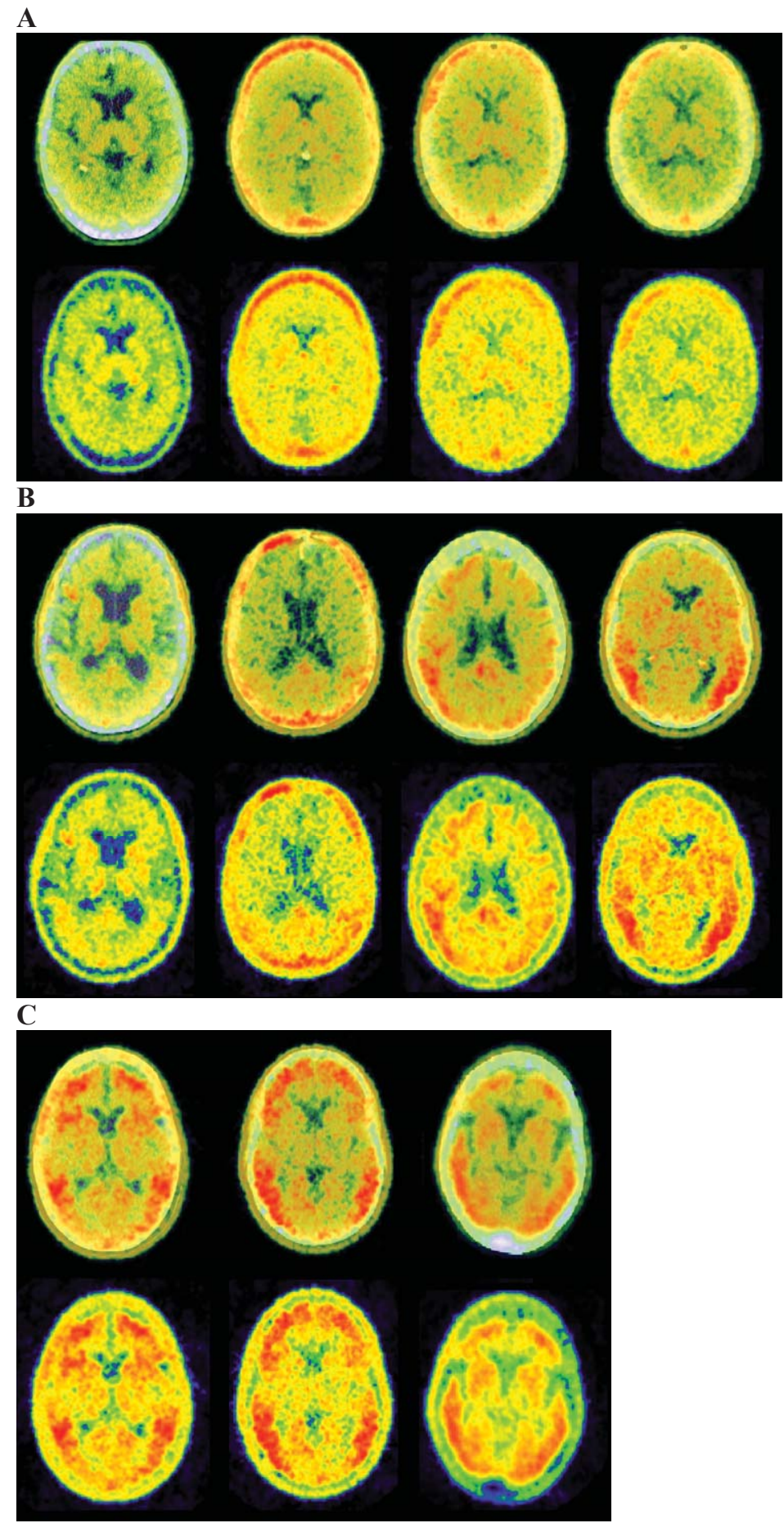

Fig. 4. A) Axial images from 30 to 50 min post injection (from left to right) of $\mathrm{HC}$ subject \#0401 (MMSE = 30), HC subject \#0402 (MMSE = 30), $\mathrm{HC}$ subject \#0404 (MMSE $=30$ ), and AD subject \#0405 (MMSE = 12). The top row shows PET/CT fusion images; the bottom row shows PET only images. B) Axial images from 30 to 50 min post injection of subjects with mild AD (from left to right) \#0413 (MMSE = 24), \#0412 (MMSE = 24), \#0410 (MMSE = 23), and \#0411 (MMSE = 24). The top row shows PET/CT fusion images; the bottom row shows PET only images. C) Axial images from 30 to 50 min post injection of subjects with moderate and severe AD (from left to right) \#0414 (MMSE = 19), \#0406 (MMSE = 14), and \#0407 (MMSE = 3). The top row shows PET/CT fusion images; the bottom row shows PET only images. 


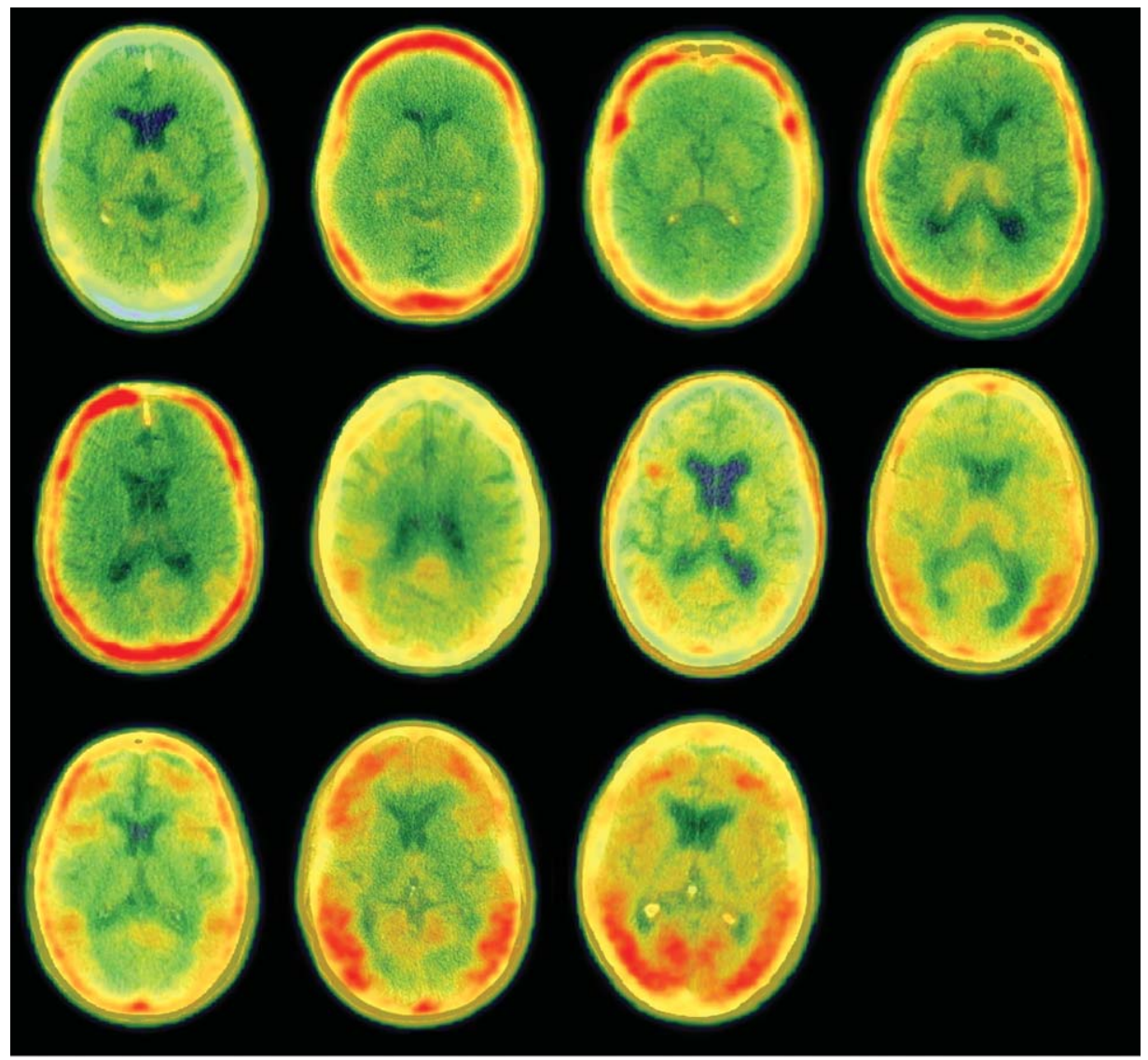

Fig. 5. Axial PET/CT fusion images from 80 to 100 min post injection. The top row from left to right shows images of HC subject \#0401, HC subject \#0402, HC subject \#0404, and AD subject \#0405. The middle row from left to right shows images of mild AD subjects \#0412, \#0410, $\# 0413$, and \#0411. The bottom row from left to right shows images of moderate and severe AD subjects \#0414, \#0406, and \#0407.

respectively) and hippocampal area $(\mathrm{SUVR}=1.22$ and 1.32 , respectively).

The occipital lobes had smaller SUVRs than the temporal lobes and hippocampal regions for the $\mathrm{AD}$ subjects, except for subject \#0407, whose radiotracer retention in the mesial temporal lobe and hippocampus (SUVR = 1.32) was equal.

For all subjects, there was low white matter retention with SUVRs $\leq 1.11$ at 30 to 50 min images with SUVRs ranging from 0.66 to 1.11 .

\section{0 to 100 min summed static images (Figure 5,} Table 3)

The late static images acquired from 80 to $100 \mathrm{~min}$ revealed low radiotracer retention with SUVR values below 1.10 for all cortical regions in the $\mathrm{HC}$ subjects and subject \#0405 (MMSE = 12).

In comparison, subjects with mild AD (MMSE 23-24) had a range of mild to moderate levels of radiotracer retention, involving the hippocampal area (SUVR = 1.28-1.42), mesial temporal lobe (SUVR = $1.12-1.41)$, lateral temporal lobe $(\mathrm{SUVR}=1.22-1.45)$, and parietal lobe (SUVR $=1.03-1.47)$. These SUVR values are similar to the $30-50 \mathrm{~min}$ summed images suggesting little change between the mid and late time points. However, there was mild radiotracer retention in the frontal lobe of subject \#0412 (SUVR = 1.25) at the late timpoint as compared to the 30-50 min images ( $S U V R=0.97)$. The increased radiotracer retention in this subject was minor compared to subjects with moderate or severe AD (SUVR=1.44-1.75). The other subjects with mild AD did not have increased radiotracer retention to suggest PHF-tau deposits in the frontal lobes (SUVR $\leq 1.11$ ). There were low levels of retention by the occipital lobes for subjects with mild AD (SUR = 1.03-1.24).

Similar to the $30-50 \mathrm{~min}$ imaging time point, there was increased radiotracer retention in the frontal lobes of subjects with moderate and severe $\mathrm{AD}$ at 
80-100 min. For subject \#0414 (MMSE = 19), there was high radiotracer retention in the frontal lobe $(\mathrm{SUVR}=1.75)$, lateral temporal lobe $(\mathrm{SUVR}=1.73)$, and parietal lobe $(\mathrm{SUVR}=1.66)$, and moderate retention in the hippocampal area $(\mathrm{SUVR}=1.49)$ and mesial temporal lobe (SUVR=1.47). For subjects with more severe $\mathrm{AD}$, the pattern of radiotracer retention was similar but with higher levels of radiotracer retention. For subject \#0406 (MMSE = 14), there was elevated radiotracer retention in the lateral temporal lobes $(\mathrm{SUVR}=1.92)$, parietal lobes (SUVR $=1.79)$, frontal lobes $(\mathrm{SUVR}=1.79)$, and moderate radiotracer retention in the mesial temporal lobes $(S U V R=1.47)$ and hippocampal area (SUVR =1.32). Similarly, severe AD subject \#0407 $(\mathrm{MMSE}=3)$ had increased radiotracer retention in the lateral temporal lobes $(\mathrm{SUVR}=2.24)$, parietal lobes $(\mathrm{SUVR}=2.05)$, frontal lobes $(\mathrm{SUVR}=1.66)$, and moderate retention in the mesial temporal lobes $($ SUVR $=1.39)$ and hippocampal area $($ SUVR $=1.45)$. The SUVR in the occipital lobes of severe AD subject \#0407 (SUVR = 1.74) was significantly higher than other moderate to severe AD subjects \#0406 $(\mathrm{SUVR}=1.11)$ and \#0414 $(\mathrm{SUVR}=1.49)$.

The 80 to $100 \mathrm{~min}$ SUVRs for the different brain regions were higher than the 30 to 50 min time range as a result of continued faster washout from the cerebellum. However, the absolute SUVmean values were very low at 80 to $100 \mathrm{~min}$ attributable to a combination of both slow washout and radioactivity decay. The cerebellum SUV mean averaged for all 11 subjects was 0.77 (range $1.30-0.57$ ) at 30 to $50 \mathrm{~min}$ and 0.50 (range $0.91-0.38$ ) at 80 to $100 \mathrm{~min}$.

As seen in the dynamic and summed images, there was low background binding in the white matter in the static images for all subjects with SUVRs $<0.9$. Additionally, there was moderate activity in the bone of the skull, but the low level of activity did not impact the data analysis of the AD subjects.

\section{DISCUSSION}

The dynamic image analyses of [F18]-T808 across both $\mathrm{HC}$ and $\mathrm{AD}$ subjects demonstrated rapid radiotracer distribution throughout the brain, rapid clearance from the cerebellum, minimal non-specific binding, and high targeted cortical radiotracer retention. These are favorable kinetic properties for a tau-targeting PET imaging agent.

The cerebellum SUV peaked at 4 min post injection for all subjects, suggesting a rapid radiotracer distri- bution profile. Separation of cerebellum TAC from the elevated cortical regions began between 7 to $10 \mathrm{~min}$ post injection for the $\mathrm{AD}$ subjects. There was a clear separation of TACs for all cortical regions from the cerebellum prior to $30 \mathrm{~min}$ post injection. This rapid clearance profile is similar to previously published amyloid radiotracers [C-11]-PiB and [F18]-florbetapir $\left(\right.$ Amyvid $\left.^{\circledR}\right)[13,14]$. The continual increase of SUVRs from 0 min through 60 min in $\mathrm{AD}$ subjects is consistent with persistent [F18]-T808 retention by the cortex and washout from the cerebellum and other non-target brain regions. There was minimal white matter retention in the brain (SUVR <1.0), in contrast to what is typically observed with several amyloid-targeting radiotracers [13, 14]. This high target-to-background cortical brain retention and low non-specific binding may facilitate the detection of even small amounts of PHF-tau in AD brains.

The kinetics and pattern of [F18]-T808 retention by the 96-year-old dementia subject \#0405 was strikingly similar to what was observed with the healthy control subjects (subject \#0401,\#0402, and \#0404), and different from the other AD subjects. A possible explanation for this discrepancy might be an incorrect clinical diagnosis of $\mathrm{AD}$. Evidence supporting this possibility is drawn from the poor association between the pathological features of $\mathrm{AD}$ and dementia in older subjects as compared to younger subjects [15]. From the available imaging data, this particular subject's low resolution, non-contrast CT from the PET/CT scan revealed diffuse cerebral atrophy with enlargement of the cortical sulci and increased ventricle size, yet there was no clear evidence of stroke or changes to differentiate dementia due to very old age versus AD. However, the subject's neurologist reported a decline of 7 points on MMSE testing over 10 years, which is a slower rate of decline than typically observed in AD patients. There are several diagnostic testing methods that can be used to increase the diagnostic certainty of $\mathrm{AD}$, including cognitive testing (MMSE, FAST), CSF (amyloid- $\beta$, tau), and neuroimaging ([F18]-FDG PET/CT, Amyvid ${ }^{\circledR}$ PET/CT, structural MRI). However, the only definitive diagnosis of $\mathrm{AD}$ can be made by postmortem brain tissue staining of both amyloid plaques and PHF-tau deposits. In the current study, subjects that were placed in the AD group only had a clinical diagnosis from their physician, based on an MMSE score of $<24$ on their first visit. The inclusion criteria were neither extensive nor definitive due to the nature of this first-in-man trial. Therefore, a subject with other causes of dementia may carry a diagnosis of AD with a qualifying MMSE score and be placed in the AD group. This uncertainty 
[18F]-T808 SUVR at 30-50 minutes post injection

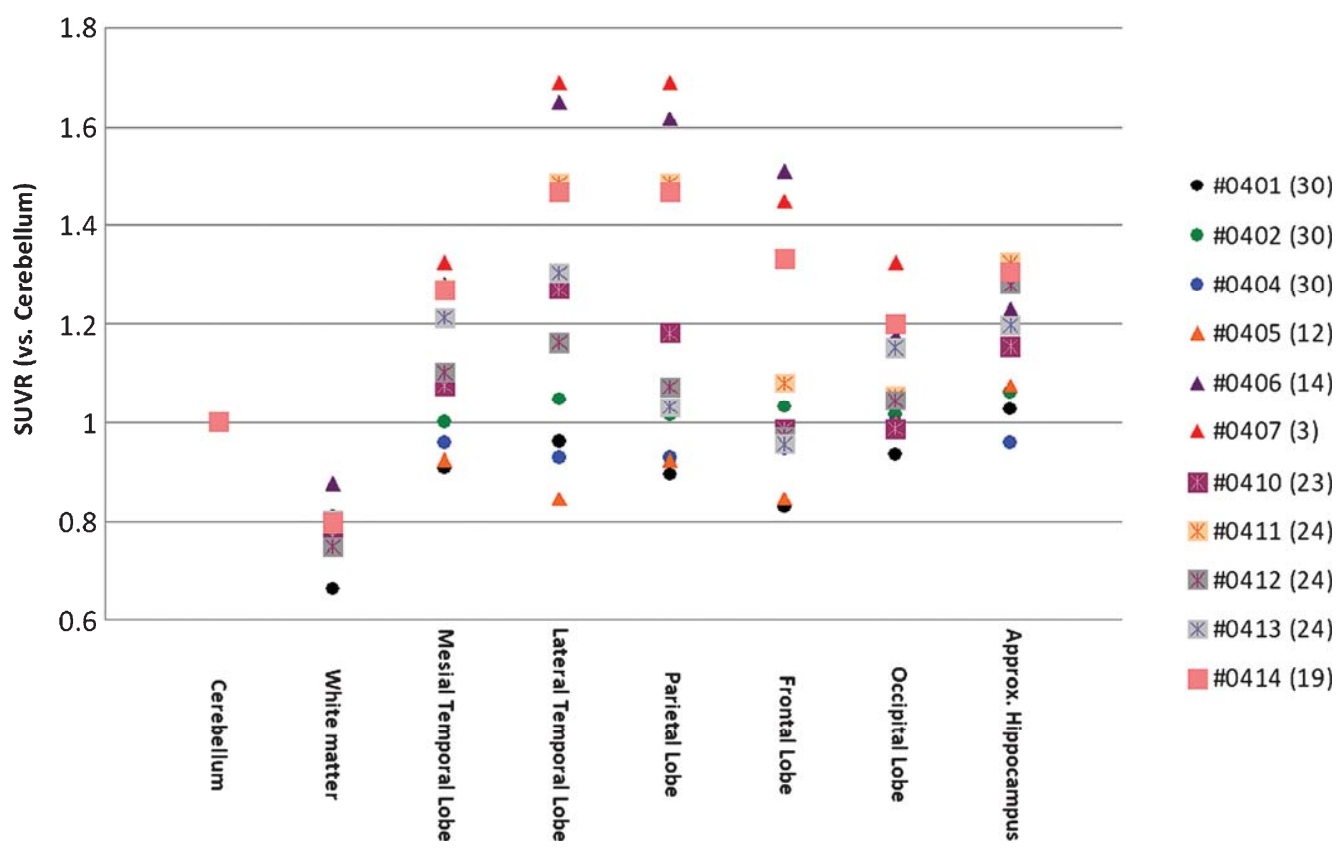

Fig. 6. Target-to-cerebellum SUV ratios of different brain regions. SUVR values determined from 30 to 50 min post injection PET data showed that more severely demented subjects had higher values across more brain regions than less severely demented subjects or healthy subjects, with one exception being patient \#0405.

illustrates the importance of developing a specific diagnostic biomarker for accurate AD diagnosis.

A review of the 30 to $50 \mathrm{~min}$ summed images and the static image from 80 to $100 \mathrm{~min}$ reveals a pattern of consistently higher cortical [F18]-T808 retention by $\mathrm{AD}$ subjects as compared to the subjects in the HC group across the temporal lobes, parietal lobes, frontal lobes, and hippocampal areas. Subjects \#0406 (MMSE= 14), \#0407 (MMSE = 3), and \#0414 (MMSE = 19) had very low MMSE scores suggestive of severe and moderate $\mathrm{AD}$, while subjects \#0410 (MMSE = 23), \#0411 (MMSE= 24), \#0412 $(\mathrm{MMSE}=24)$, and \#0413 (MMSE = 24) had MMSE score suggestive of mild AD. Comparatively, the pattern and intensity of [F18]-T808 retention correlates to the expected PHF-tau deposition seen in later stages of $\mathrm{AD}[9,10]$. Furthermore, more severely demented subjects had higher SUVR values across more brain regions than less severely demented subjects or healthy subjects (Fig. 6). At both time points, the cortical radiotracer retention in healthy subjects and white matter retention in all subjects were very low. These observations suggest that [F18]-T808 is a promising new biomarker for detecting tau deposits in AD subjects.

Further evaluation of the 4 subjects with mild AD $(\mathrm{MMSE}=23-24)$ suggests the possibility of identi- fying those with more extensive PHF-tau deposition seen in higher Braak staging [9, 10]. Subjects \#0410, $\# 0412$, and \#0413 had mild and moderate [F18]-T808 retention in the temporal lobes $(\mathrm{SUVR}=1.08-1.30$ at $30-50 \mathrm{~min} ; 1.12-1.41$ at $80-100 \mathrm{~min}$ ) and hippocampal areas $(\mathrm{SUVR}=1.15-1.28$ at $30-50 \mathrm{~min}$; $1.22-1.42$ at $80-100 \mathrm{~min}$ ), but low SUVRs in the parietal lobes (SUVR $=1.15-1.18$ at 30-50 min; $1.03-1.20$ at $80-100 \mathrm{~min})$. Comparatively, subject \#0411 had moderate [F18]-T808 retention in the temporal lobe $(\mathrm{SUVR}=1.27-1.49$ at $30-50 \mathrm{~min} ; 1.31-1.45$ at $80-100 \mathrm{~min}$ ) as well as in the parietal lobe (SUVR $=1.49$ at $30-50 \mathrm{~min}$ and 1.47 at $80-100 \mathrm{~min}$ ). PHF-tau deposits seen by pathological staining in the temporal lobe without involvement of parietal lobe is classified as Braak stage IV, while tau progression involving the parietal lobe is seen in more severe disease of Braak stage V $[9,10]$.

Compared to the subjects with mild AD, subject \#0414 (MMSE = 19) had more extensive [F18]-T808 retention in the frontal lobe at $30-50 \mathrm{~min}$ and 80-100 $\min$ (SUVR $=1.33$ and 1.75, respectively). Furthermore, subject \#0414 had similar or higher levels of [F18]-T808 retention at both 30-50 min and 80-100 min in the mesial temporal lobe, hippocampal area, lateral temporal lobe, and parietal temporal 
lobes as compared to the subjects with mild AD. This involvement of the frontal lobe is also suggestive of more severe AD as seen in Braak stage V $[9,10]$.

Subject \#0406 (MMSE = 14) had similar [F18]T808 retention in the mesial temporal lobe, hippocampal area, and frontal lobe as subject \#0414 (MMSE = 19), but also had an increased SUVR in the lateral and parietal lobe that is suggestive of increased PHF-tau deposit density. The highest [F18]T808 retention was found in the lateral temporal lobe at 30 to $50 \mathrm{~min}$ and 80 to $100 \mathrm{~min}$ (SUVR $=1.64$ and 1.92 , respectively). This was followed by the parietal lobe (SUVR $=1.60$ and 1.79 , respectively) and the frontal lobe (SUVR $=1.50$ and 1.79 , respectively), which are known to be affected only in the late Braak stages $\mathrm{V}$ and VI $[9,10]$. Even though PHF-tau accumulation has been described by Braak to initiate in the mesial temporal lobe and hippocampus, these regions are likely to develop extensive sclerosis in the later stages of the disease. Thus, the relatively lower [F18]-T808 retention seen across the time points in the mesial temporal lobe (SUVR $=1.28$ and 1.47 , respectively) and hippocampal area (SUVR $=1.22$ and 1.32 , respectively) may be due to the presence of less viable brain tissue for [F18]-T808 accumulation.

Similarly, subject \#0407 (MMSE=3) also had high levels of [F18]-T808 retention at both time points in the lateral temporal lobe (SUVR $=1.68$ and 2.24 , respectively) and parietal lobe $(\mathrm{SUVR}=1.68$ and 2.05, respectively). However, subject \#0407 had a slightly higher radiotracer retention than subject \#0406 in the parietal lobe seen on the 30 to $50 \mathrm{~min}$ images (SUVR $=1.68$ and 1.60 , respectively) and 80 to 100 min images (SUVR $=2.05$ and 1.79), which suggests progression into the latest Braak stages V-VI [9, 10]. Interestingly, subject $\# 0407$ had moderate [F18]T808 retention in the occipital lobe $(\mathrm{SUVR}=1.32$ at $30-50 \mathrm{~min} ; 1.74$ at $80-100 \mathrm{~min}$ ) that was slightly higher as compared to all other subjects having no retention to mild retention (SUVR $=0.99-1.20$ at $30-50 \mathrm{~min}, 1.11-1.24$ at $80-100 \mathrm{~min}$ ). Key neuropathology pathology studies found that the occipital lobe was unaffected at Braak stage IV but breached the parastriate and striate areas at Braak stage VI [9]. This is consistent with findings that subject \#0407 had a higher [F18]-T808 retention in the occipital lobe than all the other subjects. This subject's neurologist (author A.J.L.) stated that the subject has well known variant of $\mathrm{AD}$, which presents with complex visual disturbances consistent with posterior cortical atrophy (PCA). There are several publications utilizing a panel of AD biomarker to demonstrate PCA as a vari- ant of AD [16-19]. Furthermore, there is postmortem pathological evidence that NFTs are present in higher amounts in Brodmann areas 17 and 18 in PCA cases [20]. The next highest region of radiotracer uptake was in the frontal lobe (SUVR $=1.44$, at $30-50 \mathrm{~min}$, and 1.66 at $80-100 \mathrm{~min}$, respectively), which is consistent with a late Braak stage [9, 10]. Like subject \#0406, there was less radiotracer retention in the mesial temporal lobe (SUVR $=1.32$, at $30-50 \mathrm{~min}$, and 1.51 at 80-100 min, respectively) and the hippocampal area (SUVR $=1.32$ at $30-50 \mathrm{~min}$, and 1.45 at $80-100 \mathrm{~min}$, respectively), most likely due to sclerosis in these late stages of AD.

All subjects displayed little white matter radiotracer retention, with SUVR values $\leq 1.11$ at 30 to $50 \mathrm{~min}$ and SUVRs $<0.90$ at 80 to $100 \mathrm{~min}$. The radiotracer's low non-specific binding resulted in high contrast PET imaging. The observed white matter binding is significantly lower than what is typically observed with [F18]-labeled amyloid targeting PET radiotracers [13, 14], which may improve the gray-to-white matter contrast in the brain for more accurate activity quantitation.

There was slow radiotracer accumulation in the bone, which is consistent with [F18]-deflourination observed in our preclinical studies [11]. This bone uptake increased over time presumably as a function of [F18] formation and accumulation. At early time points such as 30 to $50 \mathrm{~min}$ post injection, the low-level bone uptake did not interfere with image interpretation. However, the total dose radiation to the bone marrow in the whole body may be elevated due to free [F18] uptake. This radiation dose will be investigated in a subsequent radiation dosimetry study.

While it is difficult to determine the optimal imaging time point to identify $\mathrm{HC}$ from $\mathrm{AD}$ subjects from a limited number of subjects in an exploratory clinical study, the current dataset does provide insight into key imaging timeframes. The results suggest that imaging at a 30 to $50 \mathrm{~min}$ timeframe sufficiently differentiates HC from AD subjects based on SUVR differences. Higher overall SUVR values were observed at the later imaging time point of 80 to $100 \mathrm{~min}$ as compared to 30 to $50 \mathrm{~min}$, which may be better suited for detecting smaller amounts of PHF-tau deposits. One must be careful not to extend the imaging time point too long after tracer injection as the SUVs in both the target and background region will steadily decrease due to tracer clearance and radioactive decay, leading to unwanted variation from scan to scan, as well as higher bone uptake that can ultimately scatter into target regions. A dedicated imaging optimization study will help define an optimal imaging time 
point as the basis for identifying patients with PHF-tau burden.

Higher SUVs for subject \#0405 were observed in all VOIs in the brain, including the target regions, nontarget regions, and the reference region when compared against the other subjects. We observed a tendentious relationship between age and SUV suggesting that an age-related component may impact the uptake and washout of the tracer, but no definitive relationship has been established. A future study with arterial blood draw and whole body scans to further evaluate the tracer kinetics with compartmental modeling, biodistribution, and clearance to better understand the pharmacokinetics will be conducted. This study will establish an optimal means for SUV quantitation and imaging time protocol in the presence of a competing [F18]-defluorination and subsequent bone uptake.

\section{CONCLUSION}

The initial analysis of [F18]-T808 clinical PET scans of 11 subjects in this first-in-man study show promise for the in vivo imaging of PHF-tau in $\mathrm{AD}$ subjects. The radiotracer displays favorable distribution and uptake kinetics with a rapid delivery into the brain and clearance from non-target tissues. These results suggest the possibility of brain imaging as early as $30 \mathrm{~min}$ after injection. All subjects showed low tracer retention in the cerebellum and white matter. In addition, the $\mathrm{HC}$ subjects showed little radiotracer retention in the cortical gray matter. The activity in bone resulting from [F18]-deflourination is separated from the cortical brain matter and does not interfere with the image evaluation, especially at early time points. More severely demented subjects generally showed higher tracer retention across more brain regions than less severely demented or HC subjects, suggestive of binding to PHF-tau deposits, with one exception being a 96-year-old dementia patient who did not show increased radiotracer retention. Additional clinical studies will further evaluate these early findings and optimize both the imaging protocol and activity quantitation.

\section{ACKNOWLEDGMENTS}

The authors will like to thank Professor Carl W. Cotman and his team at the Alzheimer's Disease Research Center for spearheading the enrollment efforts at University of California, Irvine. The authors would also like to acknowledge Beatriz Yanez, R.N., for coordi- nating Clinical Research between UCI and Siemens, as well as Drs. Hong Fan and Umesh Gangadharmath for their supplemental preparation of [F18]-T808. The research was supported entirely by internal funding of Siemens Medical Solutions USA, Inc.

Authors' disclosures available online (http://www.jalz.com/disclosures/view.php?id=1850).

\section{SUPPLEMENTARY MATERIAL}

Supplementary table, figure and text are available in the electronic version of this article: http://dx.doi. org/10.3233/JAD-130098.

\section{REFERENCES}

[1] Alzheimer's, Association (2012) Alzheimer's Association Update. Alzheimers Dement 6, 86-88.

[2] Arriagada PV, Growdon JH, Hedley-Whyte ET, Hyman BT (1992) Neurofibrillary tangles but not senile plaques parallel duration and severity of Alzheimer's disease. Neurology 42, 631-639.

[3] Buchhave P, Minthon L, Zetterberg H, Wallin K, Blennow K, Hansson O (2012) Cerebrospinal fluid levels of $\beta$-amyloid $1-42$, but not of tau, are fully changed already 5 to 10 years before the onset of Alzheimer dementia. Arch Gen Psychiatry 69, 98-106.

[4] Shao X, Carpenter GM, Desmond TJ, Sherman P, Quesada CA, Fawaz M, Brooks AF, Kilbourn MR, Albin RL, Frey KA, Scott PJH (2012) Evaluation of [11C]N-methyl lansoprazole as a radiopharmaceutical for PET imaging of tau neurofibrillary tangles. ACS Med Chem Lett 3, 936-941.

[5] Villemagne V, Furumoto S, Fodero-Tavoletti M, Harada R, Mulligan RS, Kudo Y, Masters CL, Yanai K, Rowe CC, Okamura N (2012) The challenges of tau imaging. Future Neurol 7, 409-421.

[6] Watanabe H, Ono M, Kimura H, Matsumura K, Yoshimura M, Okamoto Y, Ihara M, Takahashi R, Saji H (2012) Synthesis and biological evaluation of novel oxindole derivatives for imaging neurofibrillary tangles in Alzheimer's disease. Bioorg Med Chem Lett 22, 5700-5703.

[7] Villemagne V, Furumoto S, Fodero-Tavoletti M, Mulligan R, Hodges J, Kudo Y, Masters C, Yanai K, Rowe C, Okamura $\mathrm{N}$ (2012) In vivo tau imaging in Alzheimer's disease. J Nucl Med 53(Suppl 1), 36.

[8] Chien DT, Bahri S, Szardenings AK, Walsh JC, Mu F, Su MY, Shankle WR, Elizarov A, Kolb HC (2013) Early clinical PET imaging results with the novel PHF-tau radioligand [F-18]T807. J Alzheimers Dis 34, 457-468.

[9] Braak H, Alafuzoff I, Arzberger T, Kretzschmar H, Del Tredici K (2006) Staging of Alzheimer disease-associated neurofibrillary pathology using paraffin sections and immunocytochemistry. Acta Neuropathol 112, 389-404.

[10] Braak H, Braak E (1991) Neuropathological staging of Alzheimer-related changes. Acta Neuropathol 82, 239-259.

[11] Zhang W, Arteaga J, Cashion DK, Chen G, Gangadharmath U, Gomez LF, Kasi D, Lam C, Liang Q, Liu C, Mocharla VP, Mu F, Sinha A, Szardenings AK, Wang E, Walsh JC, Xia C, Yu C, Zhao T, Kolb HC (2012) A highly selective and specific PET tracer for imaging of tau pathologies. J Alzheimers Dis 31, 601-612. 
[12] Folstein MF, Folstein SE, McHugh PR (1975) Mini-mental state: A practical method for grading the cognitive state of patients for the clinician. J Psychiatr Res 12, 189-198.

[13] Wong DF, Rosenberg PB, Zhou Y, Kumar A, Raymont V, Ravert HT, Dannals RF, Nandi A, Brašić JR, Ye W, Hilton J, Lyketsos C, Kung HF, Joshi AD, Skovronsky DM, Pontecorvo MJ (2010) In vivo imaging of amyloid deposition in Alzheimer's disease using the novel radioligand [18F]AV-45 (Florbetapir F 18). J Nucl Med 51, 913-920.

[14] McNamee RL, Yee SH, Price JC, Klunk WE, Rosario B, Weissfeld L, Ziolko S, Berginc M, Lopresti B, DeKosky S, Mathis CA (2009) Consideration of optimal time window for Pittsburgh compound B PET summed uptake measurements. J Nucl Med 50, 348-355.

[15] Savva GM, Wharton SB, Ince PG, Forster G, Matthews FE, Brayne C (2009) Medical Research Council Cognitive Function, Ageing, Study Age, neuropathology, and dementia. $N$ Engl J Med 360, 2302-2309.

[16] Beaufils E, Dufour-Rainfray D, Hommet C, Brault F, Cottier JP, Ribeiro MJ, Mondon K, Guilloteau D (2013) Confirmation of the amyloidogenic process in posterior cortical atrophy: Value of the A $\beta 42 / \mathrm{A} \beta 40$ ratio. J Alzheimers Dis 33, 775-780.
[17] Seguin J, Formaglio M, Perret-Liaudet A, Quadrio I, Tholance Y, Rouaud O, Thomas-Anterion C, Croisile B, Mollion H, Moreaud O, Salzmann M, Dorey A, Bataillard M, Coste MH, Vighetto A, Krolak-Salmon P (2011) CSF biomarkers in posterior cortical atrophy. Neurology 76, 1782-1788.

[18] Formaglio M, Costes N, Seguin J, Tholance Y, Le Bars D, Roullet-Solignac I, Mercier B, Krolak-Salmon P, Vighetto A (2011) In vivo demonstration of amyloid burden in posterior cortical atrophy: A case series with PET and CSF findings. J Neurol 10, 1841-1851.

[19] de Souza LC, Corlier F, Habert MO, Uspenskaya O, Maroy R, Lamari F, Chupin M, Lehéricy S, Colliot O, Hahn-Barma V, Samri D, Dubois B, Bottlaender M, Sarazin M (2011) Similar amyloid- $\beta$ burden in posterior cortical atrophy and Alzheimer's disease. Brain 134, 2036-2043.

[20] Tang-Wai DF, Graff-Radford NR, Boeve BF, Dickson DW, Parisi JE, Crook R, Caselli RJ, Knoopman DS, Petersen RC (2004) Clinical, genetic, and neuropathologic characteristics of posterior cortical atrophy. Neurology 63, 1168-1174. 